\title{
CONSTRUCTION SEQUENCE SIMULATION OF A PRACTICAL SUSPEN-DOME IN JINAN OLYMPIC CENTER
}

\author{
Zhi-qiang $\mathrm{Li}^{1}$, Zhi-hong Zhang ${ }^{2,}$, Shi-lin Dong ${ }^{3}$ and Xue-yi Fu ${ }^{4}$ \\ ${ }^{1}$ Department of Building Engineering, Tongji University, Shanghai 200092, China \\ ${ }^{2}$ College of Civil Engineering, Shanghai Normal University, Shanghai 200234, China \\ ${ }^{3}$ Zhejiang University, Hangzhou 310027, China \\ 4 China Construction (Shenzhen) Design International, Shanghai 200235, China \\ *(Corresponding author: E-mail: zhangzh007@yahoo.com.cn)
}

Received: 8 March 2011; Revised: 7 July 2011; Accepted: 15 July 2011

\begin{abstract}
Construction sequence simulation analysis of a practical suspen-dome of Jinan Olympic Center is discussed in the present paper. Suspen-dome is a kind of advanced large-span space structure which is composed of an upper single-layer latticed shell and a lower cable-bar system. It is also a kind of hybrid system due to a combination of cable elements, bar elements and beam elements. Construction errors and temperature fluctuation are of importance and need to be taken into consideration during the construction process. The construction error of the structure is simulated by using the random imperfection modal method. Using the integral structure model including the concrete supporting system, the effect of the temperature variation on the suspen-dome is analyzed. The construction of suspen-dome is a little complex due to the interaction between the upper single-layer latticed shell and the lower cable-bar system. The configurations of the structure during the construction could be determined by an inverse analysis method or a mixed analysis method depending on the stages adopted in each construction scheme. And the influence to the lower cable-bar system caused by the setting-up errors at nodes is studied on the basis of one feasible construction scheme. Some meaningful and universal conclusions are given out.
\end{abstract}

Keywords: Suspen-dome, Latticed shell, Cable-bar system, Hybrid space structure, Construction errors, Temperature changes, Construction sequence simulation, Inverse analysis method, Mixed analysis method

\section{INTRODUCTION}

Structural design of the spherical suspen-dome with a diameter of $122 \mathrm{~m}$ was carried out in 2006 . This multi-purpose gymnasium locates in Jinan City, China. The arena has an approximate architectural area $60,000 \mathrm{~m}^{2}$ which can accommodate more than 12,000 audiences for the local Olympic Games held in 2009. Suspen-dome with a diameter of $122 \mathrm{~m}$ and a rise of $12.2 \mathrm{~m}$ is chosen as the roof of this gymnasium (Figures 1 and 2). The finite element model of the upper single-layer reticulated shell consists of two-node beam element. The section of the two-node beam element is a circular thin-wall steel pipe with a diameter of $377 \mathrm{~mm}$, and thickness of $14 \mathrm{~mm}$ or $16 \mathrm{~mm}$. A Sunflower-Kiewitt hybrid lattices is adopted for the upper spherical single-layer reticulated shell. Three hoops of cable-bars of Geiger type are adopted as the lower tensile system. Details of some typical joints are shown in Figures 3 to 5, such as support joint on the ring beam and two types of cable-struts joints.

Suspen-dome could be regarded as a cable-dome with the ridge cables substituted by a single-layer latticed shell. Compared to cable dome, suspen-dome is more rigid and can resist greater external loads. Further more, suspen-dome usually has a better flexibility to boundary conditions than cable dome. The structure is a kind of advanced hybrid space structures composed of beam elements, bar elements and cable elements [1]. The suspen-dome with a diameter of $122 \mathrm{~m}$ of the gymnasium of Jinan Olympic Center is the largest in the world at present. A rise to span ratio of less than 0.15 is recommended by Kitipornchai [2].

Recent work mostly focuses on the structural properties of the suspen-dome and the design of pre-tension. The static and dynamic properties of the suspen-dome are enhanced compared with the corresponding single-layer latticed shell [3]. Parametric analyses of the factors affect the buckling 
carrying capacity of the structure have been conducted. Also some design methods of pre-tension have been discussed by many researchers [4]. Unfortunately, the construction sequence simulation analysis has not been adequately investigated. More than ten suspen-dome roofs have been fabricated in the world especially in Asia. However, construction sequence simulation of cable-strut systems may be beneficial to the suspen-dome for the existence of the lower cable-bar system is to stiffen the upper single-layer latticed shell. Only implementing pre-tension to the diagonal cables, only implementing pre-tension to the hoop cables and only adjusting the length of struts are three basic methods for the construction of cable-dome [5]. But there are many difficulties in construction sequence simulation analysis of cable-dome for the coupling of mechanism displacement and elastic deformation during the construction. Nonlinear flexibility method was studied in the analysis of erection process of cable-dome by Luo [6]. However, it is not proper for the large-scale space structure because of the non-symmetric of the stiffness matrix. By controlling the original length of cables during the construction process, nonlinear finite element method was used to construction sequence simulation analysis of cable-dome by Shen [7]. Dynamic relaxation method was adopted to simulate the construction process of cable-struts by Zhang [8]. Because of the variation of geometry and component stress during the configuration process, it is pointed out by Chen [9] that only the equilibrium state of the structure after the completion of a construction stage should be considered. Inverse analysis method was proposed by Yuan [10] to simulate the construction of cable-domes step by step. However, the aforementioned studies are subject to the construction of cable dome but not for the suspen-dome. An experiment carried out by Li [11] confirmed that the suspen-dome could be constructed by implementing pre-tension to the hoop cables. Without consideration of the construction errors Li [12] simulated the construction process of a suspen-dome by a direct analysis method.

Construction sequence simulation analysis of suspen-dome is a little complex due to the interaction between the upper single-layer latticed shell and the lower cable-bar system. Actually, a considerably degree of error may be introduced if an inappropriate approach is applied to complete the analysis. Two kinds of simulation analysis methods are proposed in the present paper without any computational problems. The configuration of the structure during each construction step is determined by different methods depending on the construction stages being proceeded. Construction errors and temperature changes are of importance and need to be taken into consideration. On the basis of a practical suspen-dome roof of Jinan Olympic Center, the imperfection sensitivity and other factors effective are addressed. Construction errors of the structure are simulated. Random Imperfection Modal Method, in which a randomly generated imperfection samples subjected to a normal distribution, is applied. Using the integral structure model including the concrete supporting system for a more rational FEM analysis, the effect of temperature variation on the cable-bar system is investigated.

\section{FORCE FINDING ANALYSIS}

The lattice of the upper single-layer reticulated shell of this practical suspen-dome is combined with the Kiewitt style inside and the Lamella style outside (Figure 1). The span of the structure is $122 \mathrm{~m}$ with a rise-to-span ration of $1 / 10$. The shape of the lower cable-bar system is rib-ring type. Three hoops of cable-bar system are designed to improve the structural performance. The suspen-dome is pin-jointed on the lower reinforced concrete ring beam with the perimeter nodes being restrained in all directions. 


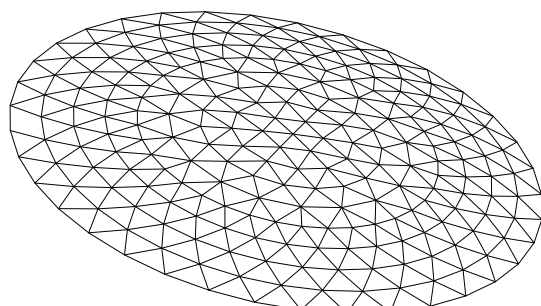

Latticed shell

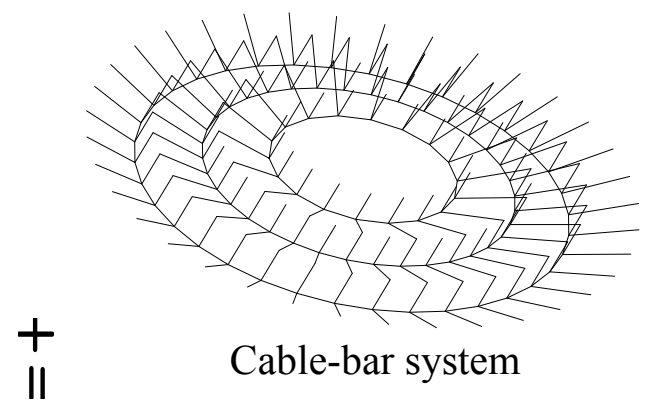

$+$

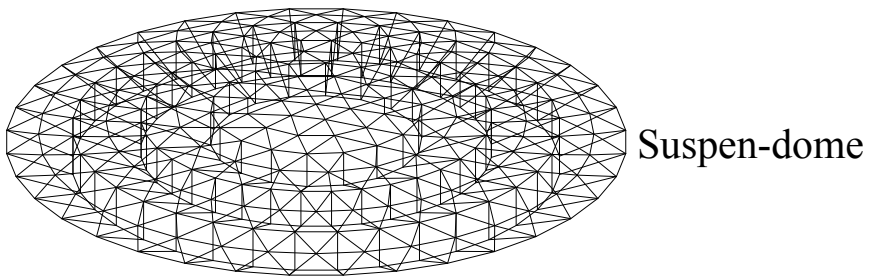

Figure 1. Suspen-dome

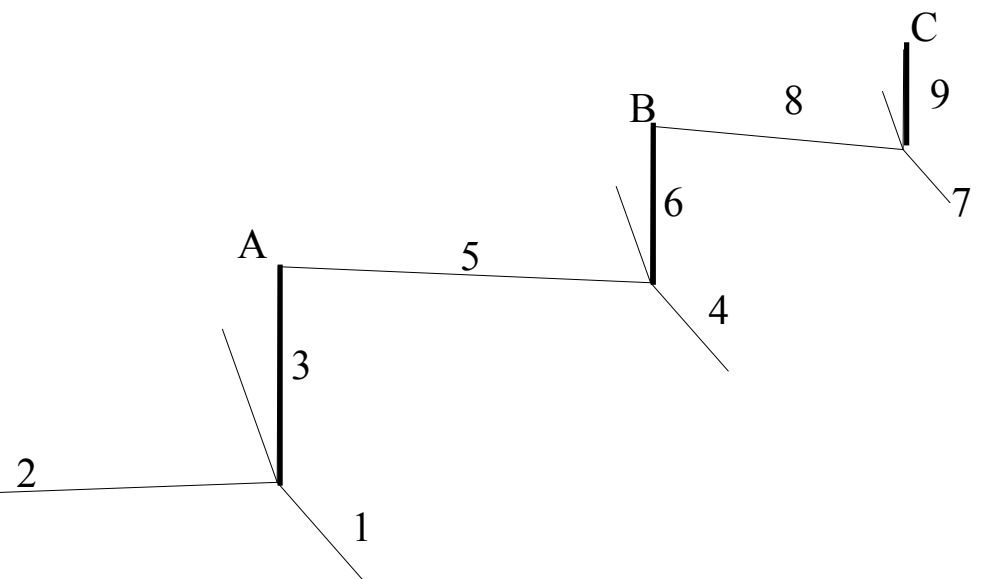

Figure 2. Group Number of Cable-struts

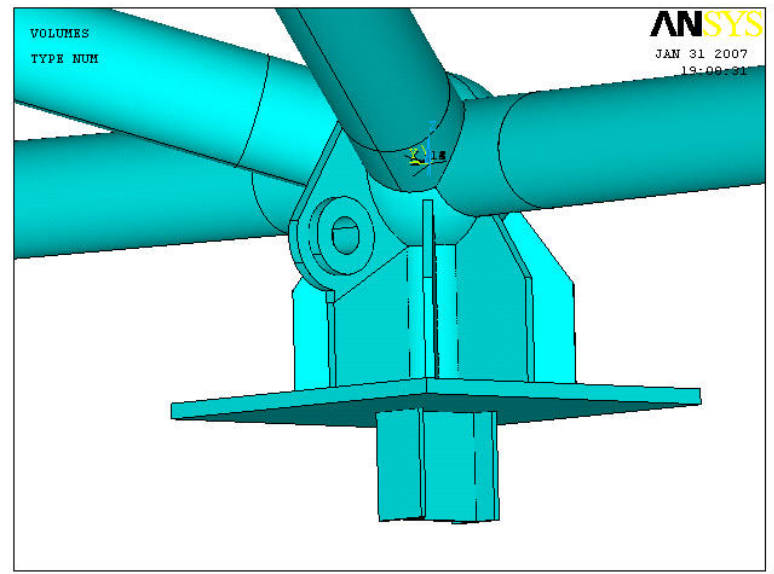

Figure 3. Support Joint 


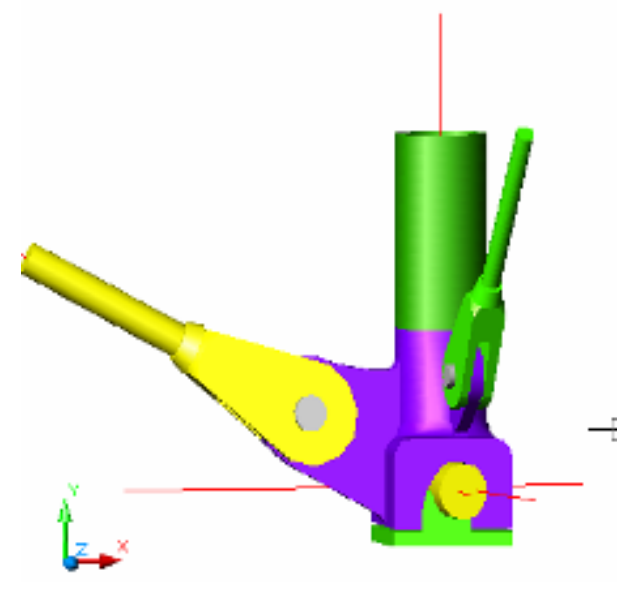

Figure 4. Cable-strut Joint

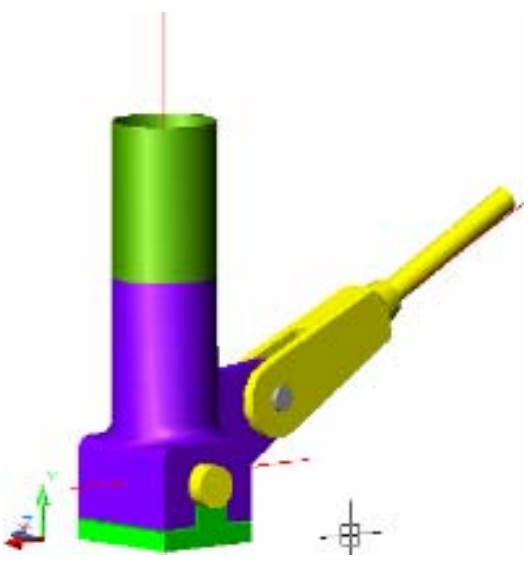

Figure 5. Cable-strut Joint

Table 1. Force Finding Analysis Results without Consideration of Self-weight (Units: kN)

\begin{tabular}{ccc}
\hline Member group & Section & Pre-tension \\
\hline 1 & $\varnothing 7 \mathrm{X} 253$ & $3.85798760 \mathrm{E}+03$ \\
2 & $\varnothing 90$ & $7.48215617 \mathrm{E}+02$ \\
3 & $\varnothing 273 \times 10$ & $-3.27996133 \mathrm{E}+02$ \\
4 & $\varnothing 5 \mathrm{X} 187$ & $1.45776145 \mathrm{E}+03$ \\
5 & $\varnothing 55$ & $2.82717311 \mathrm{E}+02$ \\
6 & $\varnothing 245 \mathrm{X} 10$ & $-1.23935110 \mathrm{E}+02$ \\
7 & $\varnothing 5 X 61$ & $4.58668838 \mathrm{E}+02$ \\
8 & $\varnothing 45$ & $1.77230854 \mathrm{E}+02$ \\
9 & $\varnothing 219 \mathrm{X} 10$ & $-7.76928916 \mathrm{E}+01$ \\
\hline
\end{tabular}

Figure 2 shows a typical frame of the lower cable-bar system. Typical nodes A, B and C are the top points of the compressive bars, and node D (not shown in Figure 2) is the central node of the upper single-layer latticed shell. The pre-tension force of the lower cable-bar system could be found according to local analysis method [13-14]. Considering to improve the structural characteristic of the upper single-layer latticed shell and to reduce the reaction force of the bearings due to a small rise-to-span ratio, the optimized pre-tension distribution is listed in Table 1.

\section{PARAMETRIC ANALYSIS OF THE SUSPEN-DOME}

\subsection{Boundary Conditions Effects On The Single-layer Latticed Shell}

Either pin-joints or rigid-joints could be applied to be the boundary joints. But at the viewpoint of design and fabrication, structures with the boundary joints being pinned are more widely used in the actual situation for the difficulty of ideal rigid-joint assumption. The effect of different boundary conditions on the upper single-layer latticed shell is shown in Table 2. The vertical displacement at node $\mathrm{D}$ named $U_{D}$ is almost the same under different boundary conditions. The vertical and radial horizontal reaction forces of the bearings named $R_{H}$ and $R_{V}$ are very close. However, the moment of the bearings could not be neglected when the suspen-dome is rigidly-jointed with ring-beam. The pin-jointed assumption is applied to the following analysis. 
Table 2. The Effect of Different Supporting Conditions

\begin{tabular}{cccc}
\hline & Pin-jointed & Rigid-jointed & Difference \\
\hline$U_{D}(\mathrm{~mm})$ & -14.128 & -14.749 & $4.40 \%$ \\
$R_{V}(\mathrm{kN})$ & $2.15 \mathrm{E}+02$ & $2.15 \mathrm{E}+02$ & $0.00 \%$ \\
$R_{H}(\mathrm{kN})$ & $4.88 \mathrm{E}+02$ & $4.77 \mathrm{E}+02$ & $-2.25 \%$ \\
\hline
\end{tabular}

\subsection{Construction Errors Effects On The Single-layer Latticed Shell}

A normal distribution assumption is applied to the nodal spatial position imperfection. The effect of construction errors on the single-layer latticed shell is listed in Table 3, which is investigated by random imperfection modal method. The maximum deviation ratio of the reaction force $R_{R}$ is confined in approximately $5 \%$ when the maximum construction error is less than $0.15 \mathrm{~m}$. The vertical displacement of node $\mathrm{D}$ named $U_{D}$ does not change too much as the maximum construction error increases.

Table 3. The Effect of Construction Errors on the Latticed Shell

\begin{tabular}{ccc}
\hline Maximum error & $U_{D}(\mathrm{~mm})$ & $R_{R}$ \\
\hline $30 \mathrm{~mm}$ & -14.381 & $1.23 \%$ \\
$60 \mathrm{~mm}$ & -14.759 & $1.72 \%$ \\
$90 \mathrm{~mm}$ & -14.625 & $1.61 \%$ \\
$120 \mathrm{~mm}$ & -15.404 & $3.72 \%$ \\
$150 \mathrm{~mm}$ & -15.644 & $4.41 \%$ \\
\hline
\end{tabular}

\subsection{The Equilibrium State of Suspen-dome Fabricated}

The single-layer latticed shell is firstly installed. The suspen-dome woks without cladding/facility appended after the cable-bar system tensioned. The vertical displacement of the node $\mathrm{D}$ named $U_{D}$ is $-0.030427 \mathrm{~m}$. The horizontal and vertical reaction force of bearings $R_{H}$ and $R_{V}$ are approximately $9.78 \times 10^{5} \mathrm{~N}$ and $2.50 \times 10^{5} \mathrm{~N}$ respectively. With consideration of the self-weight and live load during the normal state, the axial internal-force of the cable-bar system is shown in Table 4.

Table 4. The Axial Force of Cable-bar System (Kn)

\begin{tabular}{cc}
\hline Member group & Axial force $(\mathrm{kN})$ \\
\hline 1 & 3778.186 \\
2 & 732.857 \\
3 & -306.308 \\
4 & 1413.329 \\
5 & 274.624 \\
6 & -115.922 \\
7 & 449.795 \\
8 & 173.747 \\
9 & -72.661 \\
\hline
\end{tabular}




\subsection{Construction Errors and Temperature Effects on - Suspen-dome}

Some difference lies in the effect of construction errors and temperature variation on the suspen-dome with the boundary nodes being pin-jointed and rigidly-jointed. Without consideration of the elastic stiffness of the concrete supporting system, the effect of construction errors and temperature variation on the axial internal-force of cable-bar system is shown in Table 5 and Table 6 , respectively. the effect of construction errors and temperature variation on the displacement and reaction force is listed in Table 7 and Table 8. The value of column $R_{\mu}$ denotes the maximum deviation ratio between the mean axial internal-force and the design pre-tension among each group of cable-bar elements. The value of column $R_{\sigma}$ denotes the maximum deviation ratio between the axial internal-force standard deviation and the mean axial internal-force among each group of cable-bar elements. The value of column $R_{R H}$ describes the maximum deviation ratio of the horizontal reaction force while the value of column $R_{R V}$ is the vertical reaction force.

Table 5. Construction Error Effects on the Axial Internal-force of the Cable-bar Elements

\begin{tabular}{ccc}
\hline Max error & $R_{\sigma}$ & $R_{\mu}$ \\
\hline $30 \mathrm{~mm}$ & $1.75 \%$ & $1.17 \%$ \\
$60 \mathrm{~mm}$ & $5.05 \%$ & $1.24 \%$ \\
$90 \mathrm{~mm}$ & $9.27 \%$ & $1.29 \%$ \\
$120 \mathrm{~mm}$ & $11.42 \%$ & $1.30 \%$ \\
$150 \mathrm{~mm}$ & $18.42 \%$ & $1.43 \%$ \\
\hline
\end{tabular}

Table 6. Temperature Variation Effects on the Axial Internal-force of the Cable-bar Elements

\begin{tabular}{ccc}
\hline Temperature & $R_{\sigma}$ & $R_{\mu}$ \\
\hline$+10^{\circ} \mathrm{C}$ & $1.17 \%$ & $-13.66 \%$ \\
$+20^{\circ} \mathrm{C}$ & $1.14 \%$ & $-27.20 \%$ \\
$-10^{\circ} \mathrm{C}$ & $1.25 \%$ & $13.47 \%$ \\
$-20^{\circ} \mathrm{C}$ & $1.29 \%$ & $27.44 \%$ \\
\hline
\end{tabular}

Table 7. Construction Errors Effects on the Displacement and the Reaction Force

\begin{tabular}{cccc}
\hline Max error & $U_{D}(\mathrm{~mm})$ & $R_{R H}$ & $R_{R V}$ \\
\hline $30 \mathrm{~mm}$ & -30.37 & $1.57 \%$ & $2.46 \%$ \\
$60 \mathrm{~mm}$ & -31.297 & $4.67 \%$ & $5.66 \%$ \\
$90 \mathrm{~mm}$ & -30.933 & $4.90 \%$ & $8.14 \%$ \\
$120 \mathrm{~mm}$ & -29.932 & $6.08 \%$ & $9.08 \%$ \\
$150 \mathrm{~mm}$ & -28.835 & $7.70 \%$ & $11.04 \%$ \\
\hline
\end{tabular}

Table 8. Temperature Variation Effects on the Displacement and the Reaction Force

\begin{tabular}{cccc}
\hline Temperature & $U_{D}(\mathrm{~mm})$ & $R_{R H}$ & $R_{R V}$ \\
\hline$+10^{\circ} \mathrm{C}$ & -5.788 & $-30.13 \%$ & $0.16 \%$ \\
$+20^{\circ} \mathrm{C}$ & 58.884 & $-60.00 \%$ & $0.10 \%$ \\
$-10^{\circ} \mathrm{C}$ & -55.14 & $30.54 \%$ & $-0.29 \%$ \\
$-20^{\circ} \mathrm{C}$ & -80.142 & $60.61 \%$ & $0.10 \%$ \\
\hline
\end{tabular}


It is learned from Tables 5 to 8 that construction errors have a little effect on horizontal reaction force, but a significant effect on vertical reaction force. However, the effect of temperature variation on reaction force is on the contrary. It is also concluded that construction errors have little effect on the mean axial internal-force among each group of the cable-bar elements, but a significant effect on the standard deviation of the axial force. And the effect of temperature variation on the axial force among each group of the cable-bar elements is on the contrary. The guaranteed rate of the normal distribution between $\mu-\sigma$ and $\mu+\sigma$ is only $68.3 \%$ while between the interval $(\mu-3 \sigma, \mu+3 \sigma)$ is $99.7 \%$. Hence it is evident that maximum construction error should be under $0.03 \mathrm{~m}$ for the deviation of axial force may be limited within $5.25 \%$.

\subsection{Temperature Effects Based on the Integral Structure Analysis}

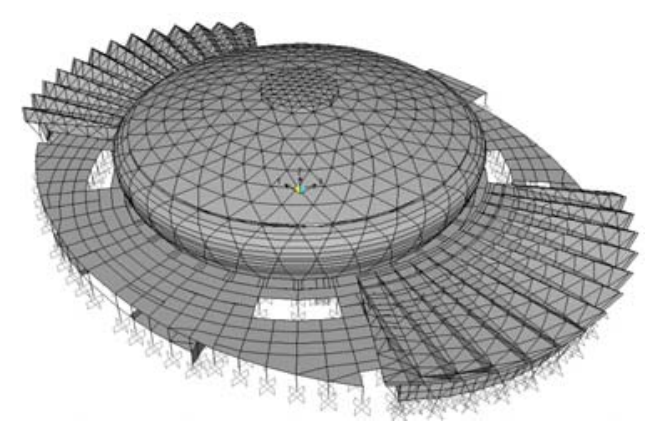

Figure 6. The Integral Model of the Gymnasium

The upper steel structure and the lower concrete supporting structure have a compatible state of deformation in real conditions. It may not be more rational to investigate the effects of temperature variation on the upper steel structure with the boundary nodes being pin-jointed without the consideration of the elastic stiffness contribution of the lower concrete supporting system. The linear thermal expansion coefficient of the concrete is very close to the steel. The effect of temperature variation on the axial force of the cable-bar element decreases while the integral structure model (Figure 6) with the lower concrete supporting system is adopted. The results are summarized in Table 9.

Table 9. Temperature Variation Effects on the Integral Model

\begin{tabular}{ccc}
\hline Temperature & $U_{D}(\mathrm{~mm})$ & $R_{\mu}$ \\
\hline$+0{ }^{\circ} \mathrm{C}$ & -9.306 & $0.00 \%$ \\
$+10^{\circ} \mathrm{C}$ & -4.944 & $-1.50 \%$ \\
$+20^{\circ} \mathrm{C}$ & -5.409 & $-3.00 \%$ \\
$-10^{\circ} \mathrm{C}$ & -16.549 & $1.50 \%$ \\
$-20{ }^{\circ} \mathrm{C}$ & -23.798 & $3.00 \%$ \\
\hline
\end{tabular}




\section{CONSTRUCTION SEQUENCE SIMULATION ANALYSIS OF SUSPEN-DOME}

The lower cable-bar system plays an important role in the resistance of the external loads. Implementing pre-tension to hoop cables and adjusting the length of struts could be used to complete the fabrication of suspen-dome. However, the method of implementing pre-tension to diagonal cables is used in the present paper. So the elements of group 2, group 5 and group 8 are used as actively-pre-tensioned cables.

Both mechanism and mechanical analysis must be carried out during the construction sequence simulations for the coupling of mechanism displacement and elastic deformation [14]. The configuration and the pre-tension force distribution of the suspen-dome vary with the construction process. The equilibrium state of the structure will be obtained if the configuration or the pre-tension distribution of the suspen-dome is determined during the construction.

Pre-tension contributes much to the increment of the ultimate carrying capacity of suspen-dome. It is noted that the configuration of the structure could be identified exactly as the pre-tension distribution is determined by nonlinear finite element analysis. The determination of the initial status without pre-tension is very difficult for the large degree of indeterminacy of the structure. The inverse analysis method could be applied to the identification of the configuration and the pre-tension distribution during the construction if a single-stage fabrication scheme is adopted, based on the state of the structure fabricated. Firstly, the state before the last step could be determined by killing elements fabricated at the last step. The rest may be deduced by analogy and the state of each step could be identified by killing elements fabricated at the next step. According to the inverse analysis method, the controlling parameters including the pre-tension and configuration of the structure in each construction step could be identified by removing the diagonal cables in sequence. Each equilibrium state during the construction could be determined. This method has an advantage of clear in conception and convenient in computation. And the ideal state of the structure given out byarchitects could be obtained if the inverse analysis method is applied to construction sequence simulation.

However, the inverse analysis method may result in a problem of high construction costsince the diagonal cables are tensioned for only once. Therefore, a multi-stage fabrication scheme may be necessary to reduce construction cost. And a mixed animation analysis method composed of inverse analysis firstly and direct analysis secondly with acceptable accuracy is proposed in the paper, while a multi-stages fabrication scheme is applied to the construction. The inverse analysis is used to obtain the intermediate state for purpose of decreasing the axial internal-force of cables at the first stage while the direct analysis is used to obtain the controlling state at the second stage. The mixed animation analysis method could be used in the engineering practice very well for better applicability and lower construction cost.

Three construction schemes are presented in details from Section 4.1 to Section 4.3.

\subsection{Scheme 1}

A single-stage fabrication scheme is adopted in this Section. Each group of diagonal cables is tensioned respectively for only one time. Tensile force at each step could be obtained in the equilibrium state by killing elements fabricated in the next step. As shown in Figure 7, the sequence of the construction in scheme 1 is as follows: 
Table 10. The Variation of the Axial Force of the Cable-bar Elements (Units: kN)

\begin{tabular}{cccc}
\hline Group & Step 1 & Step 2 & Step 3 \\
\hline 1 & 0 & 0 & 3778.186 \\
2 & 0 & 0 & $\mathbf{7 3 2 . 8 5 7}$ \\
3 & 0 & 0 & -306.308 \\
4 & 0 & 712.963 & 1413.329 \\
5 & 0 & $\mathbf{1 3 8 . 8 6 2}$ & 274.624 \\
6 & 0 & -55.821 & -115.922 \\
7 & 225.372 & 311.421 & 449.795 \\
8 & $\mathbf{8 7 . 2 3 6}$ & 120.290 & 173.747 \\
9 & -34.559 & -49.111 & -72.661 \\
\hline
\end{tabular}

Table 11. The Displacement of the Typical Nodes A, B, C and D (Units: mm)

\begin{tabular}{cccc}
\hline disp & Step 1 & Step 2 & Step 3 \\
\hline$U_{A}$ & -15.867524 & -49.227523 & 4.751852 \\
$U_{B}$ & -31.966751 & 0.914842 & 8.518034 \\
$U_{C}$ & 6.930325 & 26.713785 & 12.395205 \\
$U_{D}$ & -10.389931 & -7.598448 & -30.426954 \\
\hline
\end{tabular}

Step $1,50.2 \%$ of the designed pre-tension is imposed to the elements of group 8 (the inner hoop of diagonal cables);

Step 2, 50.56\% of the designed pre-tension is imposed to the elements of group 5 (the middle hoop of diagonal cables);

Step 3,100\% of the designed pre-tension is imposed to the elements of group 2 (the outermost hoop of diagonal cables);

The steps of the animation analysis are opposite to the sequence of construction. Hence, the inverse analysis method is adopted. The sequences of animation analysis are presented in the following text:

Step 1, the equilibrium state of the structure and the tensile force of the actively-pre-tension cables described in the aforementioned text are obtained by killing the elements of group 2 and 5;

Step 2, the equilibrium state and the tensile force of the cables of group 5 could be identified by killing the elements of group 2;

Step 3, the equilibrium state and the tensile force of the cables of group 2 are equal to the ideal fabricated state of the structure;

The variation of the axial internal-force of the cable-bar elements is shown in the Table 10, where the bold italic number denotes the controlling tensile force of the actively-pre-tension cables in each single construction step. The displacement of the structure during the construction is listed in Table 11. 


\section{Latticed shell}
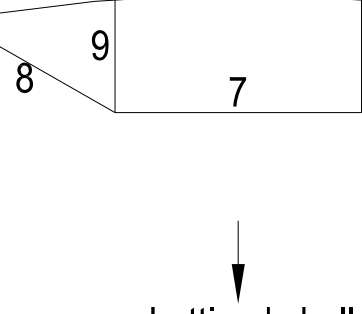

Latticed shell

5
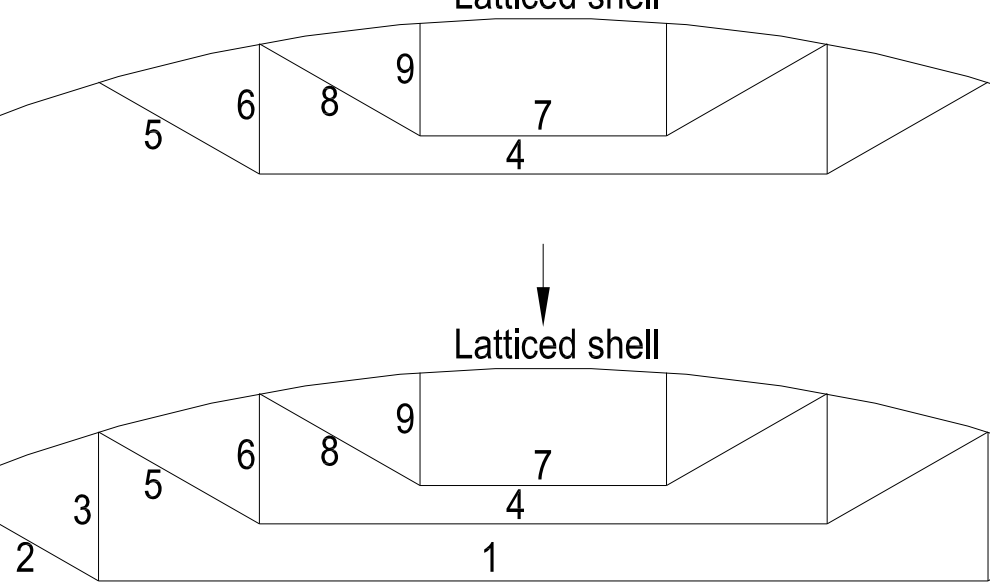

Figure 7. Construction Sequence in Scheme 1

Note that the tensile force imposed to the elements of group 5 and 8 are approximately $50.2 \%$ and $50.56 \%$ of the designed pre-tension respectively in this scheme. The largest tensile force of the actively-pre-tension cables is $732857 \mathrm{~N}$. It is shown that the nodes near to the cables being tensioned have the upward vertical displacement. The space position of each node meets the design state after the structure constructed.

\subsection{Scheme 2}
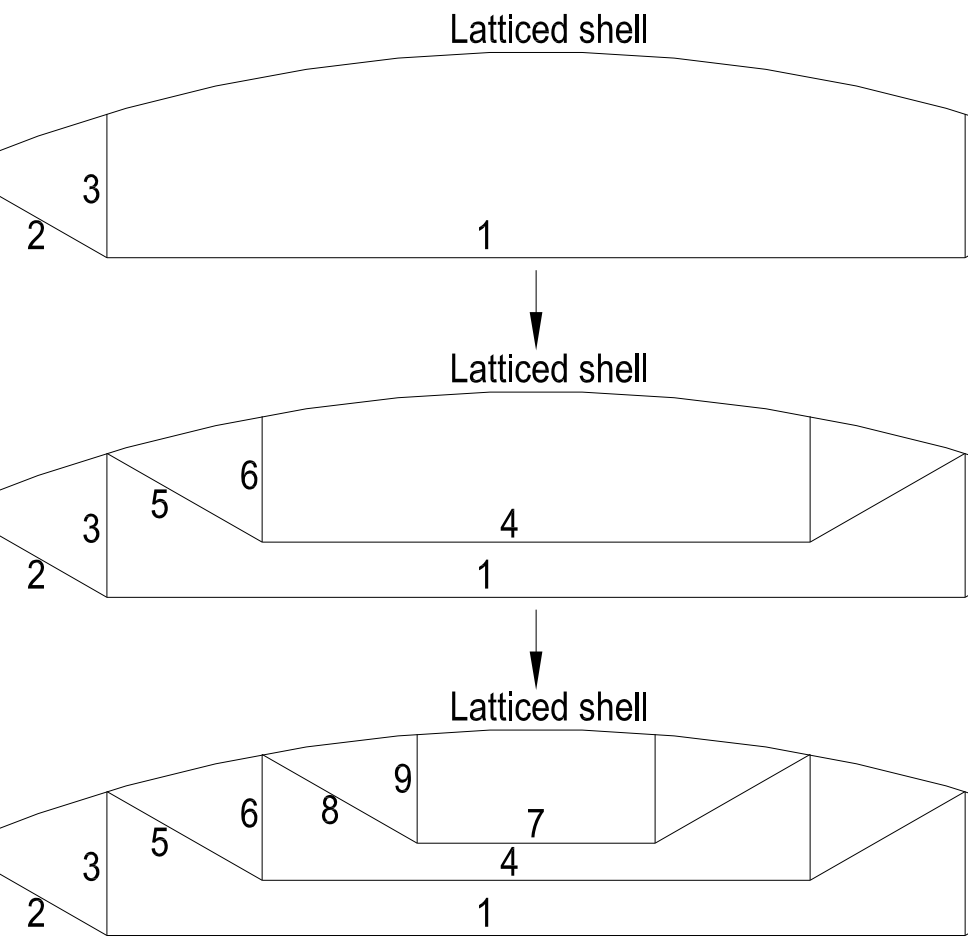

Figure 8. Construction Sequence in Scheme 2 
A single-stage fabrication method is adopted in this scheme. The diagonal cables of cable-bar system of each ring are tensioned simultaneously. Diagonal cables in different rings are tensioned respectively for only one time. Tensile force in each step could be acquired in the equilibrium state by killing elements fabricated in the next step. As shown in Figure 8, the construction steps in this scheme are as follows:

Step $1,77.78 \%$ of the design pre-tension force is imposed to the elements of group 2;

Step 2, 92.76\% of the design pre-tension force is imposed to the elements of group 5;

Step 3,100\% of the design pre-tension force is imposed to the elements of group 8;

The arrangement of this construction scheme is just on the contrary of the scheme 1 . The steps of the animation in ANSYS are opposite to the actual situation. Each configuration of the structure is determined by the inverse analysis respectively since a single-stage fabrication scheme is applied according to the aforementioned text. The sequences of animation analysis by the nonlinear finite element method in ANSYS are presented as follows:

Step 1, the equilibrium state and the tensile force of the elements of group 2 are obtained by killing the elements of group 5 and 8;

Step 2, the equilibrium state and the tensile force of the elements of group 5 are computed out by killing the elements of group 8;

Step 3, the equilibrium state and the tensile force of the elements of group 8 are just the ideal fabricated state of the structure;

Table 12. The Variation of the Axial Internal-force of the Cable-bar Elements (Units: kN)

\begin{tabular}{cccc}
\hline Group & Step 1 & Step 2 & Step 3 \\
\hline 1 & 2933.442 & 3689.205 & 3778.186 \\
2 & $\mathbf{5 7 0 . 0 4 6}$ & 715.473 & 732.857 \\
3 & -235.557 & -298.477 & -306.308 \\
4 & 0 & 1310.571 & 1413.329 \\
5 & 0 & 254.737 & 274.624 \\
6 & 0 & -107.103 & -115.922 \\
7 & 0 & 0 & 449.795 \\
8 & 0 & 0 & $\mathbf{1 7 3 . 7 4 7}$ \\
9 & 0 & 0 & -72.661 \\
\hline
\end{tabular}

Table 13. The Displacement of the Typical Nodes A, B, C and D (Units: mm)

\begin{tabular}{cccc}
\hline disp & Step 1 & Step 2 & Step 3 \\
\hline$U_{A}$ & 55.08182 & 10.113194 & 4.751852 \\
$U_{B}$ & -36.695176 & 30.941798 & 8.518034 \\
$U_{C}$ & -49.284646 & -29.192398 & 12.395205 \\
$U_{D}$ & -38.559757 & -37.318611 & -30.426954 \\
\hline
\end{tabular}


The variation of the axial internal-force of the cable-bar elements is given out in Table 12. The bold italic number denotes the controlling tensile force of the actively-pre-tension cables in each construction step. The displacement of the structure during the configuration is shown in Table 13. Note that the tensile force exerted to the elements of group 2 and 5 are approximately $77.78 \%$ and $92.76 \%$ of the designed pre-tension. The largest tensile force of actively-pre-tension cables is $570046 \mathrm{~N}$ in this scheme. It is noted that the configuration of the structure is close to the design state after the completion of the implement of the elements of group 2 and 5. It implies that the contribution of the implemented pre-tension of the elements of group 8 to the fabrication of the suspen-dome could be omitted. And the nodes near to the cables being tensioned have a positive vertical displacement. It seems that the position of each node meets the design state after the structure constructed.

\subsection{Scheme 3}

A two-stage fabrication method is adopted in this scheme. The actively-pre-tensioned cables are tensioned simultaneously in each step. Diagonal cables in different rings are tensioned respectively for twice. Tensile force of each step in stage 1 is assumed to $50 \%$ of the design pre-tension. Tensile force of each step in stage 2 could be acquired in the equilibrium state by killing elements fabricated in the next step. The arrangements of the construction are as follows:

\section{Stage 1:}

Step $1,50 \%$ of the design pre-tension is imposed to the elements of group 2; Step 2, 50\% of the design pre-tension is imposed to the elements of group 5; Step $3,50 \%$ of the design pre-tension is imposed to the elements of group 8 ;

\section{Stage 2:}

Step $1,79.44 \%$ of the design pre-tension is imposed to the elements of group 8; Step 2, 84.4\% of the design pre-tension is imposed to the elements of group 5; Step 3,100\% of the design pre-tension is imposed to the elements of group 2;

A mixed analysis method combined with the inverse analysis and direct analysis is applied to the construction sequence simulation of the structure in this scheme. Each configuration of the structure during the construction is determined by the nonlinear finite element analysis. The tensile force of the actively-pre-tension cables of group 8 and 5 should be set to approximately $79.44 \%$ and $84.4 \%$ of the designed pre-tension respectively in the stage 2 . The arrangements of the animation computation are described according to the sequences of the calculations as follows:

\section{Stage 1:}

Step 1, the equilibrium state in this step is obtained by the following three parts of analysis:

An intermediate state is obtained by the analysis of killing the elements of group 8;

A state is obtained by the analysis of killing the elements of group 5 from the latest state;

A single-point temperature stress is imposed to one end of the elements of group 2 to simulate a compressive force until the axial internal-force of the actively-pre-tensioned cables achieve approximately $50 \%$ of the design pre-tension force from the second state in this step. The temperature could be determined by some trial computations. 
Step 2, the equilibrium state in this step is obtained by the following two parts of analysis:

An intermediate state is obtained by the analysis of alive the elements of group 5 being killed from the state of step 1 firstly;

A single-point temperature stress is imposed to one end of the elements of group 5 to simulate a compressive force until the axial internal-force of the actively-pre-tension cables achieve approximately $50 \%$ of the design pre-tension from the latest state in this step. The temperature could be determined by some trial computations.

Step 3, the equilibrium state in this step is obtained by the following two parts of analysis:

An intermediate state is obtained by the analysis of alive the elements of group 8 being killed from the state of step 2 firstly;

A single-point temperature stress is imposed to one end of the elements of group 8 to simulate a compressive force until the axial internal-force of the actively-pre-tension cables achieve approximately $50 \%$ of the design pre-tension from the latest state in this step. The temperature could be determined by some trial computations.

\section{Stage 2:}

Step 1, the temperature of the end of the elements of group 8 which is used as the actively-pre-tension point in the last stage should be set to zero for the reason of energy conservation. And then the axial internal-force of the actively-pre-tension cables achieve approximately $79.44 \%$ of the design pre-tension force after the analysis completed;

Step 2, the temperature of the end of the elements of group 5 which is used as the actively-pre-tension point in the last stage should be set to zero for the reason of energy conservation. It is noted that the axial internal-force of the actively-pre-tension cables achieve approximately $84.4 \%$ of the design pre-tension force after the nonlinear analysis finished;

Step 3, the temperature of the end of the elements of group 2 which is used as the actively-pre-tension point in the last stage should be set to zero for the reason of energy conservation. Note that the axial internal-force of the cables and bars achieve approximately $100 \%$ of the design pre-tension force after the computation fulfilled;

The variation of the axial internal-force of the cable-bar elements is shown in Table 14 while the displacement of the structure during the fabrication is presented in Table 15. The bold italics number in Table 14 denotes the controlling force of the actively-pre-tensioned cable in each construction step. The tensile force of the elements of group 8 in the stage 2 is $79.44 \%$ of the design pre-tension but not $100 \%$, while those elements of group 5 in the stage 2 is $84.4 \%$ of the design pre-tension. A desired axial internal-force of each element is recorded when the last construction step completed. The nodes which are close to the actively-pre-tension cables have a positive vertical displacement. It is noted that the position of each node meets the design state after the structure constructed. A mostly economical construction method of scheme 3 could be regarded for the lowest magnitude tensile force of the actively-pre-tensioned cables in the above 3 schemes with the maximum value of $366615 \mathrm{~N}$ during the construction. Therefore, cheaper equipments could be used for the construction. In this scheme the structure rigidity is shaped gently and engineers have more chance to adjust the construction errors. 
Table 14. The Variation of the Axial Force of the Cable-bar Elements (Units: kN)

\begin{tabular}{ccccccc}
\hline Group & Step 1 & Step 2 & Step 3 & Step 4 & Step 5 & Step 6 \\
\hline 1 & 1888.494 & 2271.428 & 2315.070 & 2341.094 & 2557.788 & 3778.186 \\
2 & $\mathbf{3 6 6 . 6 1 5}$ & 440.973 & 449.479 & 454.552 & 496.647 & $\mathbf{7 3 2 . 8 5 7}$ \\
3 & -144.892 & -177.900 & -181.740 & -184.041 & -202.916 & -306.308 \\
4 & 0 & 706.163 & 757.943 & 787.603 & 1191.159 & 1413.329 \\
5 & 0 & $\mathbf{1 3 7 . 3 8 9}$ & 147.584 & 153.434 & $\mathbf{2 3 1 . 7 9 5}$ & 274.624 \\
6 & 0 & -55.117 & -59.947 & -62.760 & -97.558 & -115.922 \\
7 & 0 & 0 & 223.507 & 357.443 & 405.799 & 449.795 \\
8 & 0 & 0 & $\mathbf{8 6 . 3 8 9}$ & $\mathbf{1 3 8 . 0 2 3}$ & 156.702 & 173.747 \\
9 & 0 & 0 & -34.369 & -56.810 & -65.007 & -72.661 \\
\hline
\end{tabular}

Table 15. The Vertical Displacement of the Typical Nodes A, B, C and D (Units: mm)

\begin{tabular}{ccccccc}
\hline Disp & Step1 & Step2 & Step3 & Step4 & Step5 & Step6 \\
\hline$U_{A}$ & 27.378023 & 4.620033 & 1.992812 & 0.427138 & -12.428725 & 4.607384 \\
$U_{B}$ & -26.383106 & 7.599213 & -3.477436 & -9.949669 & 6.060556 & 8.382912 \\
$U_{C}$ & -31.769468 & -23.459562 & -3.470519 & 8.335725 & 16.886617 & 12.342352 \\
$U_{D}$ & -26.452058 & -26.218994 & -24.937856 & -24.541326 & -24.673249 & -32.994649 \\
\hline
\end{tabular}

\section{NODAL SPTIAL POSITION ERROR EFFECTS ON CONSTRUCTION}

The effect due to nodal spatial position errors on the lower cable-bar system during the construction is analyzed. Based on the Scheme 1 of fabrication, the effect of the nodal position errors is investigated by using random imperfection modal method where the randomly normal distribution imperfection samples are generated. The effects of different magnitude of nodal position errors at all construction steps are listed from Table 19 to Table 20. Since guarante rate of the normal distribution between $\mu-\sigma$ and $\mu+\sigma$ is only $68.3 \%$ while between the interval $(\mu-3 \sigma, \mu+3 \sigma)$ is $99.7 \%$, it is obvious that the maximum nodal spatial position error should be under $0.03 \mathrm{~m}$ with the deviation of axial internal-force may achieve the limited value of approximately $11.4 \%$.

Table 19. The Effect of Position Errors on the Construction Step 1

\begin{tabular}{ccc}
\hline Max error & $R_{R}$ & $R_{\sigma}$ \\
\hline $30 \mathrm{~mm}$ & $5.18 \%$ & $0.30 \%$ \\
$60 \mathrm{~mm}$ & $6.93 \%$ & $2.08 \%$ \\
$90 \mathrm{~mm}$ & $6.32 \%$ & $4.05 \%$ \\
$120 \mathrm{~mm}$ & $6.56 \%$ & $7.60 \%$ \\
$150 \mathrm{~mm}$ & $6.94 \%$ & $7.62 \%$ \\
\hline
\end{tabular}

Table 20. The Effect of Position Error on the Construction Step 2

\begin{tabular}{ccc}
\hline Max error & $R_{R}$ & $R_{\sigma}$ \\
\hline $30 \mathrm{~mm}$ & $3.24 \%$ & $3.80 \%$ \\
$60 \mathrm{~mm}$ & $5.95 \%$ & $7.05 \%$ \\
$90 \mathrm{~mm}$ & $9.85 \%$ & $9.08 \%$ \\
$120 \mathrm{~mm}$ & $10.41 \%$ & $14.90 \%$ \\
$150 \mathrm{~mm}$ & $15.84 \%$ & $20.70 \%$ \\
\hline
\end{tabular}




\section{CONCLUSIONS}

(1) Effects of different supporting conditions on the upper single layer latticed shell of the suspen-dome are not significant.

(2) Nodal spatial position errors should be restricted under an ultimate magnitude for its significant effect on suspen-dome.

(3) Little effects of construction errors on the mean axial internal-force among each group of cable-bar elements is obtained, while a great effect on the standard deviation of the axial force is obtained. And the effect of temperature variation is just on the contrary.

(4) A relatively low influence of temperature variation to the structure could be realized if integral structure model with concrete supporting system is adopted.

(5) The displacement of the structure during the construction is combined with mechanism displacement and elastic deformation. And a significant effect of the passively-pre-tension cables on the actively-pre-tensioned cables during the construction process should be much concerned.

(6) The configuration of the structure could be determined exactly as the pre-tension distribution is acquired. The inverse analysis method is suggested for the construction sequence simulation analysis of the suspen-dome if a single-stage fabrication scheme is adopted. A mixed analysis method with a combination of inverse and direct analysis proposed may be beneficial for the construction sequence simulation analysis of suspen-dome if a multi-stage- fabrication scheme is adopted.

(7) A multi-stage fabrication scheme is recommended due to low cost of construction.

\section{ACKNOWLEDGEMENTS}

The authors gratefully acknowledge the support of Leading Academic Discipline Project of Shanghai Normal University. Project Number: A-7001-12-002007.

\section{REFERENCES}

[1] Dong, S.L. and Zhao, Y., "Discussion On Types and Classifications of Spatial Structures", Journal of Civil Engineering, 2004, Vol. 37, No. 1, pp. 7-12. [In Chinese]

[2] Kitipornchai, S. and Kang, W.J. et al., "Factors Affecting the Design and Construction of Lamella Suspen-dome Systems", Journal of Constructional Steel Research, 2005, Vol. 61, pp. 764-785.

[3] Kang, W.J. and Chen, Z.H. et al., "Analysis and Design of The General and Outmost-ring Stiffened Suspen-dome Structures”, Engineering Structures, 2003, Vol. 25, pp. 1685-1695.

[4] Kawaguchi, M. and Abe, M. et al., "Design, Tests and Realization of 'Suspen-dome' System", Journal of the IASS, 1999, Vol. 40, No. 131, pp. 179-92.

[5] Zhen, J.H and Dong, S.L. et al., "Experimental Research on Construction Process of Levy Type Cable Dome”, Journal of Building Structures, 2006, Vol. 27, No. 1, pp. 112-116. [In Chinese] 
[6] Luo, Y.Z. and Shen, Y.B., "Initial Configuration Determination of Cable Dome Structure and Analysis of Its Configuration Process", Journal of Zhejiang University (Engineering Science), 2004, Vol. 38, No. 10, pp. 1321-1327. [in Chinese]

[7] Shen, Z.Y. and Zhang, L.X., "Simulation of Erection Procedures of Cable Domes Based on FEM", Chinese Journal of Computational Mechanics, 2002, Vol. 19, No. 4, pp. 466-471. [in Chinese]

[8] Zhang, Z.H. and Dong, S.L. et al., "Design and Construction Analysis of Cable-strut Tensile Structures”, Spatial Structures, 2003, Vol. 9, No. 2, pp. 20-24. [in Chinese]

[9] Chen, L.M. and Dong, S.L. et al., "Theoretical and Experimental Study on The Construction of Cable Domes", Journal of Civil Engineering, 2006, Vol. 39, No. 11, pp. 33-36. [in Chinese]

[10] Yuan, X.F. and Dong, S.L., "Inverse Analysis of Construction Process of Cable Dome", Journal of Building Structures, 2001, Vol. 22, No. 2, pp. 75-79. [in Chinese]

[11] Li, G.L. and Wang, Z.Q. et al., "Test Study on Shaping of Double Ellipse Suspen-dome", Architecture Technology, 2007, Vol. 38, No. 5, pp. 348-351. [in Chinese]

[12] Li, Y.M. and Zhang, Y.G., "Determination of Cable Forces During Construction for Cable-supported Latticed Shells", Journal of Building Structures, 2004, Vol. 25, No. 4, pp. 76-81. [in Chinese]

[13] Zhang, Z.H. and Zhang, M.S., "Discussion on The Equilibrium Matrix Theory and Static and Stability Analysis of A Hybrid Spatial Structure Composed of Cables, Bars and Beams", Engineering Mechanics, 2004, Vol. 22, No. 6, pp. 7-14. [in Chinese]

[14] Zhang, Z.H. and Zhang, M.S. et al., "Discussion on Some Problems of Beam-string Structures”, Engineering Mechanics, 2004, Vol. 21, No. 6, pp. 26-30. [in Chinese] 